Supporting Information for

\title{
Earth-Abundant Molecular Z-Scheme Photoelectrochemical Cell for Overall Water-Splitting
}

Christopher D. Windle, ${ }^{1}$ Hiromu Kumagai, ${ }^{2}$ Masanobu Higashi, ${ }^{3}$ Romain Brisse,${ }^{4}$ Sebastian Bold, ${ }^{1,5,6}$ Bruno Jousselme, ${ }^{4}$ Murielle Chavarot-Kerlidou, ${ }^{1}$ Kazuhiko Maeda, ${ }^{2}$ Ryu Abe, ${ }^{3 *}$ Osamu Ishitani, ${ }^{2 *}$ Vincent Artero*1

${ }^{1}$ Laboratoire de Chimie et Biologie des Métaux, Université Grenoble Alpes, CNRS, CEA, 17 rue des Martyrs, 38054 Grenoble, Cedex, France.

${ }^{2}$ Department of Chemistry, School of Science, Tokyo Institute of Technology, O-okayama 2-121-NE-1, Meguro-ku, Tokyo 152-8550, Japan.

${ }^{3}$ Department of Energy and Hydrocarbon Chemistry, Graduate School of Engineering, Kyoto University, Katsura, Nishikyo-ku, Kyoto 615-8510, Japan.

${ }^{4}$ Laboratory of Innovation in Surface Chemistry and Nanosciences (LICSEN), NIMBE, CEA, CNRS, Université Paris-Saclay, CEA Saclay, 91191 Gif-sur-Yvette, Cedex, France.

${ }^{5}$ Institute of Physical Chemistry and Abbe Center of Photonics, Friedrich Schiller University Jena, Helmholtzweg 4, 07743 Jena, Germany.

${ }^{6}$ Department Functional Interfaces, Leibniz Institute of Photonic Technology Jena (IPHT), Albert-Einstein-Straße 9, 07745 Jena, Germany. 

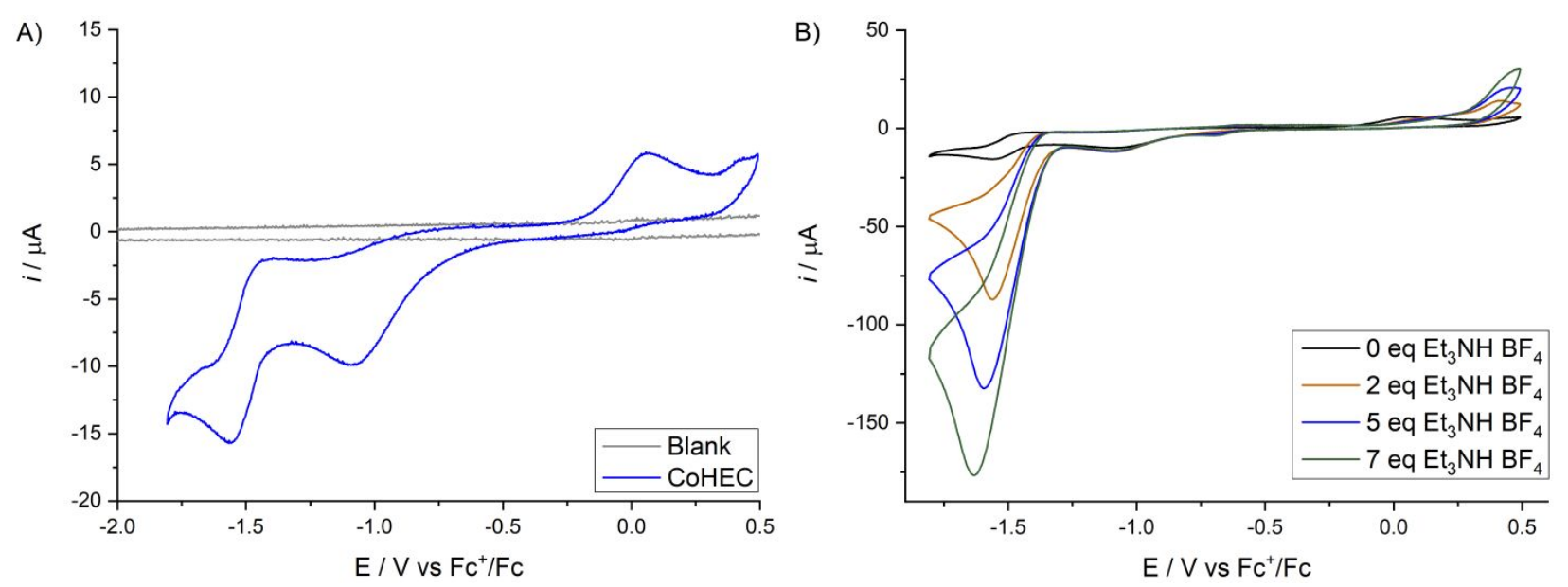

Figure S1. A) Cyclic voltammograms of CoHEC in DMF $\left.\left(0.1 \mathrm{M} \mathrm{Bu}_{4} \mathrm{NPF}_{6}\right) \mathrm{B}\right)$ with increasing amounts of $\left(\mathrm{Et}_{3} \mathrm{NH}\right) \mathrm{BF}_{4}$
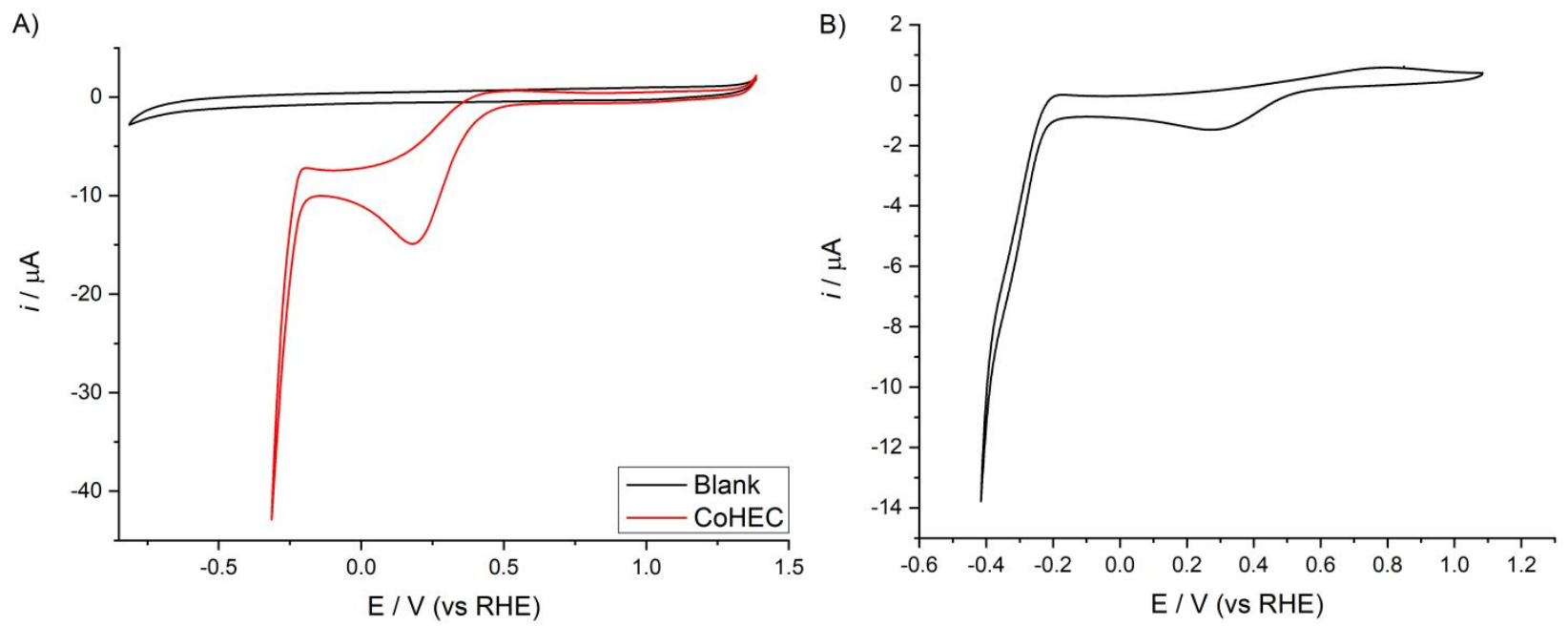

Figure S2. A) Cyclic voltammetry of CoHEC in MES buffer $\mathrm{pH} 5.5 \mathrm{~B}$ ) CoHEC in sodium phosphate buffer $\mathrm{pH} 7$ 


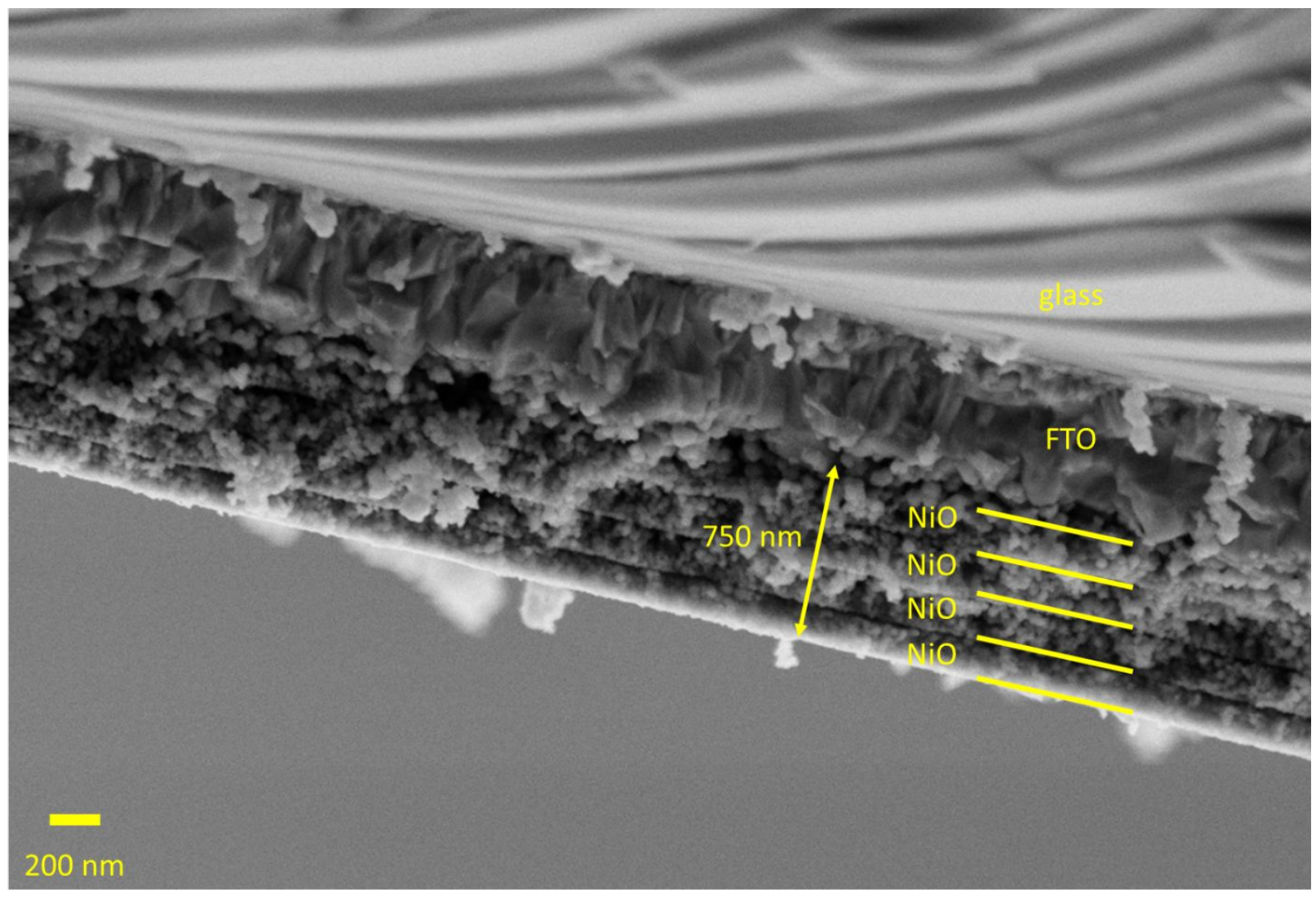

Figure S3. SEM image taken side-on of the 4-layer spin-coated $\mathrm{NiO}$ 


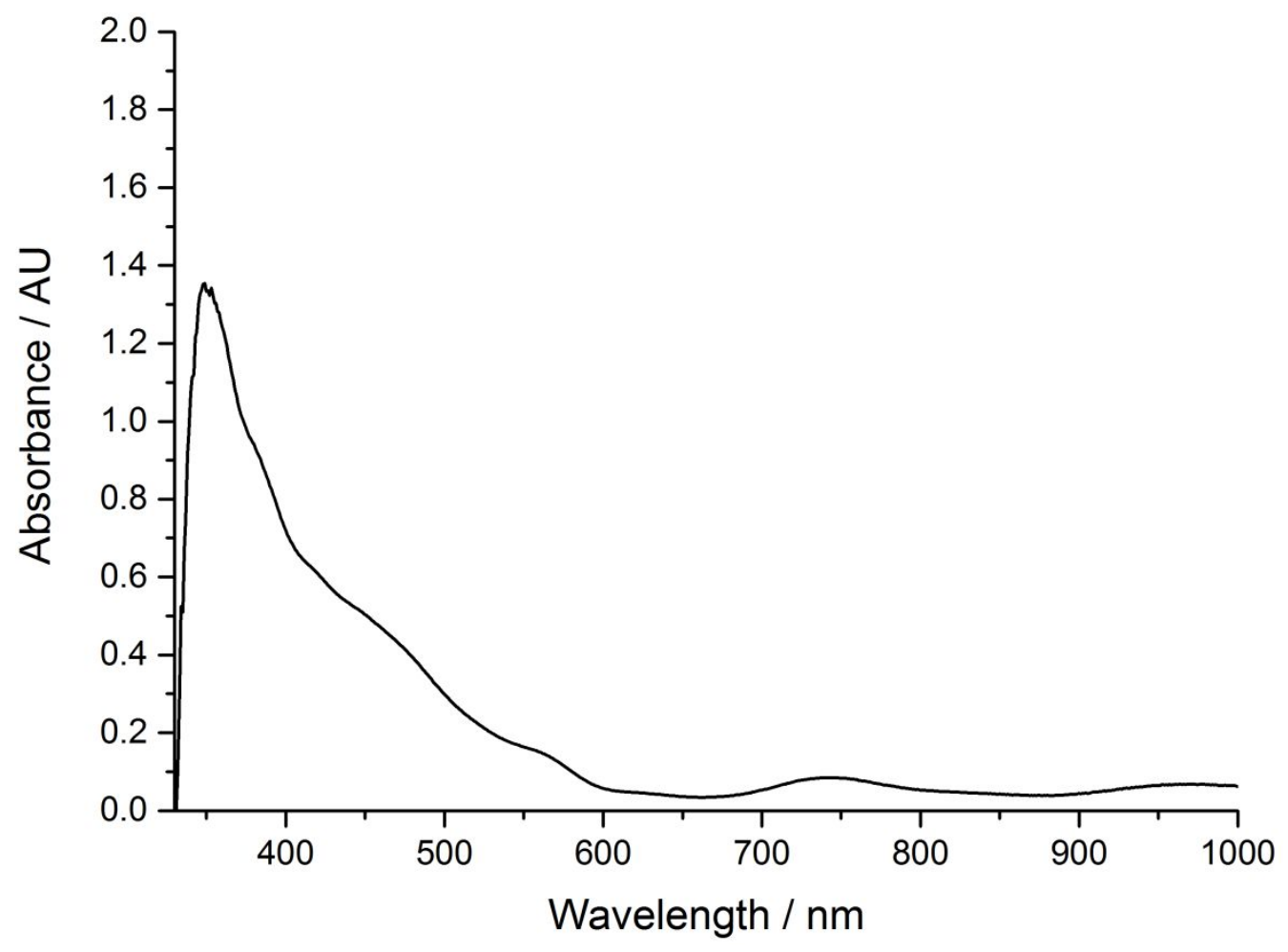

Figure S4. UV/Vis spectrum of NiOlRBG-174lCoHEC in $\mathrm{NaCl}(0.1 \mathrm{M})$ poised at $0 \mathrm{~V}$ vs $\mathrm{Ag} / \mathrm{AgCl}$. Spectrum is after subtraction of a background taken with bare $\mathrm{NiO}$ poised at $0 \mathrm{~V}$ vs $\mathrm{Ag} / \mathrm{AgCl}$.

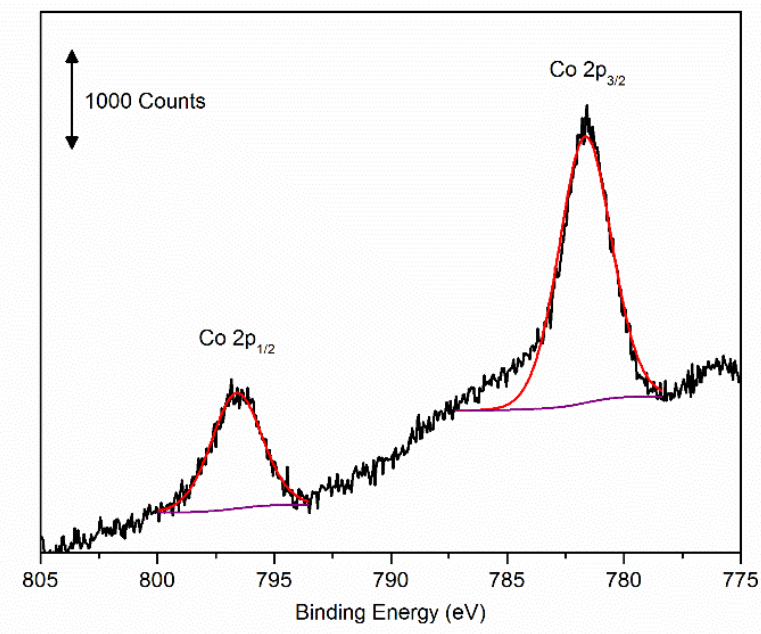

Figure S5. High resolution XPS spectrum in the cobalt region for pristine NiO|RBG-174|CoHEC. Co $2 \mathrm{p} 3 / 2$ and $2 \mathrm{p} 1 / 2$ have almost a 2:1 ratio with binding energies of $781.6 \mathrm{eV}$ and $796.5 \mathrm{eV}$ 


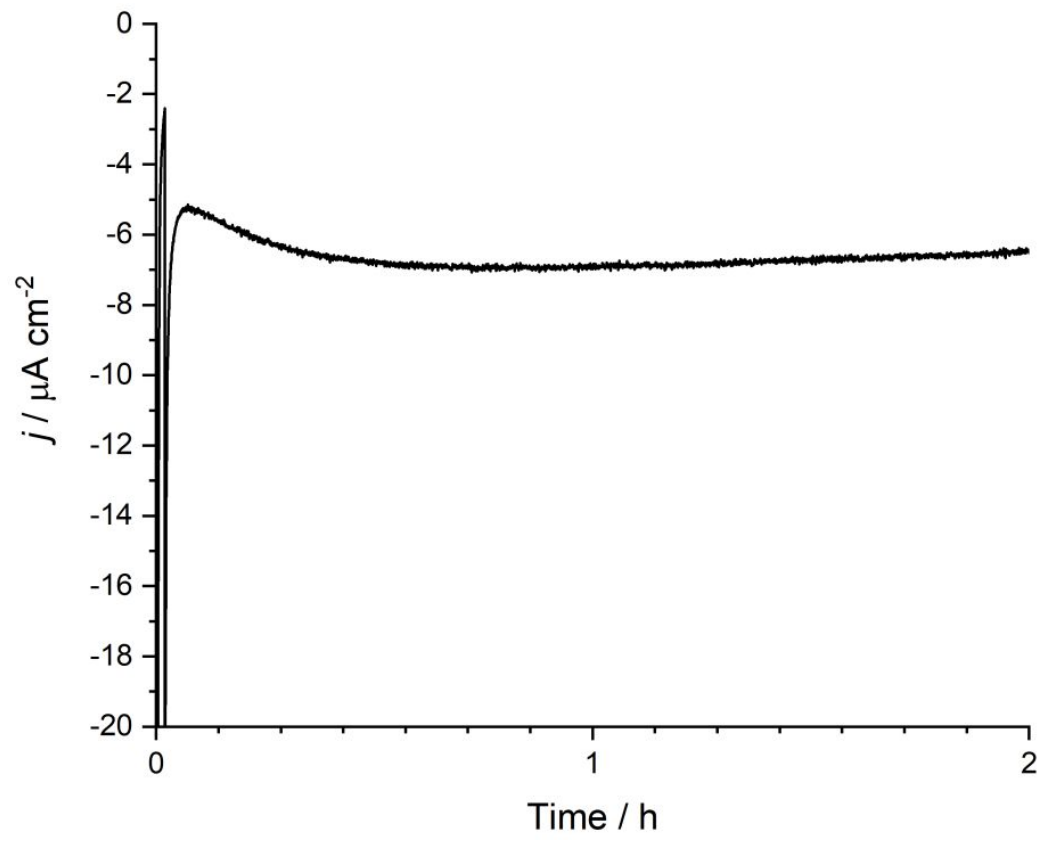

Figure S6. Chronoamperometry of NiO|RBG-174|CoHEC at $0.14 \mathrm{~V}$ vs RHE for $2 \mathrm{~h}(\lambda>400 \mathrm{~nm}$, $\left.50 \mathrm{~mW} . \mathrm{cm}^{-2}\right)$ in MES buffer (0.1 M, pH 5.5).
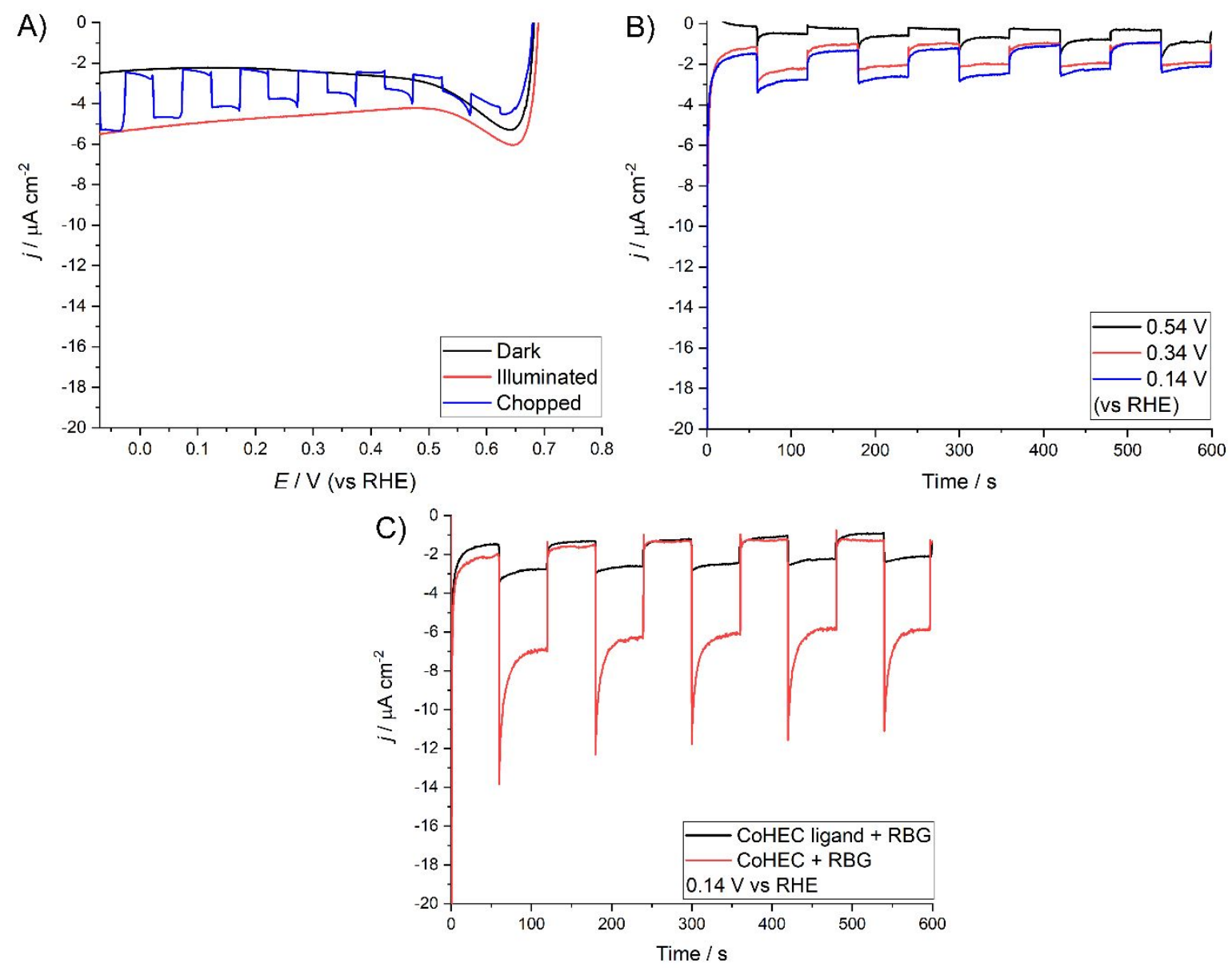
Figure S7. A) LSV of NiO|RBG-174|[4,4'-bipyridine]-2,6-dicarboxylate without (black) and with (red) illumination, and under chopped illumination (blue). B) Chronoampometry of NiO|RBG-174|[4,4'-bipyridine]-2,6-dicarboxylate under chopped illumination at $0.54 \mathrm{~V}$ (black), $0.34 \mathrm{~V}$ (red) and $0.14 \mathrm{~V}$ (blue) vs RHE. C) Comparison of photocurrent at $0.14 \mathrm{~V}$ vs RHE between NiO|RBG-174|CoHEC and NiO|RBG-174|[4,4'-bipyridine]-2,6-dicarboxylate. All in MES buffer (0.1M, pH 5.5).

A)

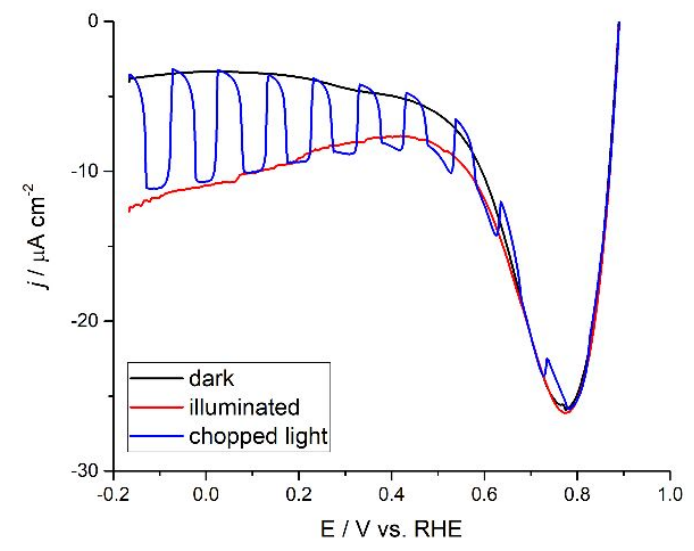

B)

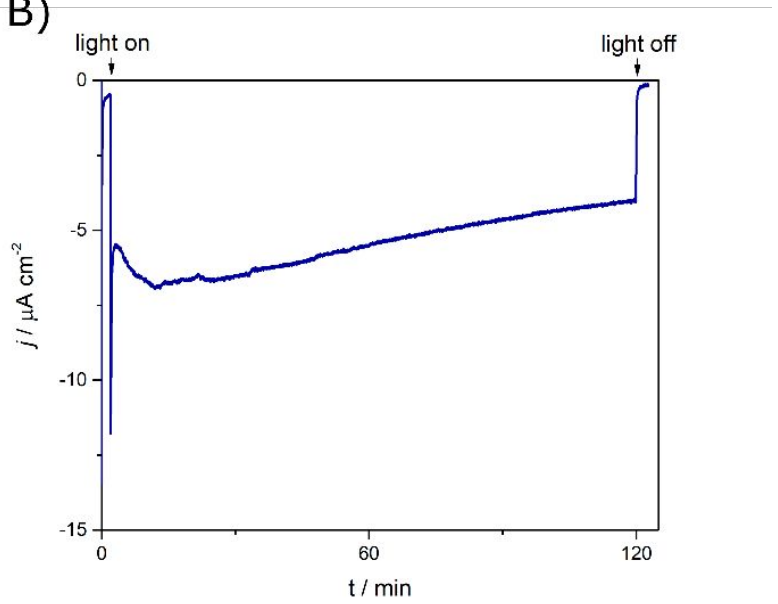

Figure S8. A) LSV of NiO|RBG-174|CoHEC without (black) and with (red) illumination, and under chopped illumination (blue). B) Chronoampometry of NiO|RBG-174|CoHEC under illumination at an applied potential of $0.14 \mathrm{~V}$ vs RHE. All in sodium phosphate buffer $\mathrm{pH} 7$. 


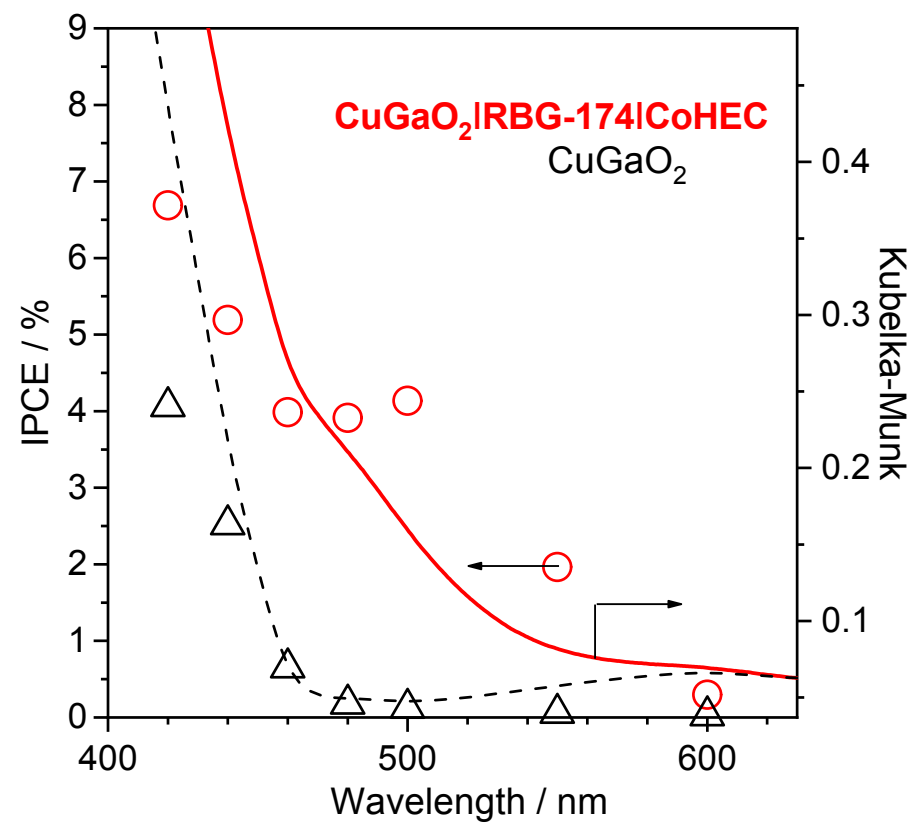

Figure S9. IPCE spectra of $\mathbf{C u G a O}_{2}|\mathbf{R B G}-\mathbf{1 7 4}| \mathbf{C o H E C}$ and bare $\mathrm{CuGaO}_{2}$ at $0.41 \mathrm{~V}$ vs $\mathrm{RHE}$ in $0.05 \mathrm{M}$ phosphate buffer $\mathrm{pH}$ 7. The diffuse reflectance spectra of these electrodes are also shown.

Table S1. Photophysical and redox properties of RBG, CoHEC, $\mathrm{NiO}$ and $\mathrm{CuGaO}_{2}$.

\begin{tabular}{|c|c|c|c|c|c|c|c|c|c|c|c|c|c|c|}
\hline & \multirow{2}{*}{$\begin{array}{l}\lambda_{\mathrm{Abs}}{ }^{\mathrm{a}} \\
/ \mathrm{nm}\end{array}$} & \multirow{2}{*}{$\begin{array}{c}\varepsilon \\
/ \mathrm{M}^{-} \\
{ }^{1} . \mathrm{cm}^{-1}\end{array}$} & \multirow{2}{*}{$\begin{array}{c}\lambda_{\mathbf{E m}} / \\
\mathbf{n m}\end{array}$} & \multicolumn{2}{|c|}{$\begin{array}{c}\mathrm{E}_{\text {red }}(\mathrm{RBG}- \\
174 / \mathrm{RBG}- \\
\left.174^{-}\right) / \mathrm{V}\end{array}$} & \multicolumn{2}{|c|}{$\begin{array}{c}E^{*} \text { red }(\text { RBG- } \\
174^{*} / \mathrm{RBG}- \\
\left.174^{-}\right) / \mathrm{V}\end{array}$} & \multicolumn{2}{|c|}{$\begin{array}{c}\text { Ered }\left(\mathbf{C o}^{\mathrm{III} / \mathrm{II}}\right) \\
/ \mathbf{V}\end{array}$} & \multicolumn{2}{|c|}{$\begin{array}{c}\text { Ered }^{\mathrm{b}} \\
\left(\mathrm{Co}^{\mathrm{II} / \mathrm{I}}\right) / \mathrm{V}\end{array}$} & \multicolumn{2}{|c|}{ Flat band / V } & \multirow{2}{*}{$\mathbf{E}_{0-0}$} \\
\hline & & & & $\begin{array}{c}\text { vs } \\
\text { NHE }\end{array}$ & $\begin{array}{c}\text { vs } \\
\mathrm{RHE}^{\mathrm{c}}\end{array}$ & $\begin{array}{c}\text { vs } \\
\text { NHE }\end{array}$ & $\begin{array}{c}\text { vs } \\
\mathrm{RHE}^{\mathrm{c}}\end{array}$ & $\begin{array}{c}\text { vs } \\
\text { NHE }\end{array}$ & $\begin{array}{c}\text { vs } \\
\mathrm{RHE}^{\mathrm{c}}\end{array}$ & $\begin{array}{c}\text { vs } \\
\text { NHE }\end{array}$ & $\begin{array}{c}\text { vs } \\
\text { RHE }^{\mathrm{c}}\end{array}$ & $\begin{array}{c}\text { vs } \\
\text { NHE }\end{array}$ & $\begin{array}{c}\text { vs } \\
\mathrm{RHE}^{\mathrm{c}}\end{array}$ & \\
\hline $\begin{array}{l}\text { RBG- } \\
174_{\mathrm{tBu}}{ }^{\mathrm{d}}\end{array}$ & 442 & 27100 & 688 & -1.15 & -0.74 & 1.23 & 1.64 & - & - & - & - & - & - & $\begin{array}{c}2.38 \\
\mathrm{eV}\end{array}$ \\
\hline СоHEC & - & - & - & - & - & - & - & -0.13 & 0.28 & -0.63 & -0.22 & - & - & - \\
\hline $\mathrm{NiO}^{\mathrm{e}}$ & - & - & - & - & - & - & - & - & - & - & - & 0.30 & 0.72 & - \\
\hline $\mathrm{CuGaO}_{2}{ }^{\mathrm{f}}$ & - & - & - & - & - & - & - & - & - & - & - & $0.67^{\mathrm{e}}$ & 1.06 & - \\
\hline
\end{tabular}

a) recorded in $\mathrm{CH}_{2} \mathrm{Cl}_{2}$ b) taken from onset of catalysis in water c) converted using RHE $=$ NHE $\left.+(0.059 \times \mathrm{pH}) \mathrm{d}\right)$ tBu-protected precursor of RBG174; data from R. Brisse et al., Sustainable Energy Fuels 2018, 2, 648 and from N. Kaeffer et al., Chem. Sci., 2018, 9, 6721 e) at pH 7 from Natu et al., ACS Appl. Mater. Interfaces 2012, 4, 5922 f) in pH 6.6 from Kumagai et al., Chem. Sci. 2017, 8 , 4242

Table S2. Free energy change of electron transfer between system components 


\begin{tabular}{|l|l|c|}
\hline Electron donor $\left(\mathbf{E}_{\mathbf{0 x}}\right)$ & Electron Acceptor $\left(\mathbf{E}_{\text {red }}\right)$ & $\Delta \mathbf{G}$ \\
\hline $\mathrm{RBG}-174^{-}$ & $\mathrm{Co}{ }^{\mathrm{III}} \mathrm{HEC}$ & $-1.02^{\mathrm{a}}$ \\
\hline $\mathrm{RBG}-174^{-}$ & $\mathrm{Co}{ }^{\mathrm{II}} \mathrm{HEC}$ & $-0.54^{\mathrm{a}}$ \\
\hline $\mathrm{NiO}$ & RBG-174* & $-0.93^{\mathrm{b}}$ \\
\hline $\mathrm{CuGaO}_{2}$ & RBG-174* & $-0.56^{\mathrm{b}}$ \\
\hline
\end{tabular}

a) Free energy change of electron transfer calculated using $\Delta \mathrm{G}=\mathrm{E}_{\mathrm{ox}}-\mathrm{E}_{\mathrm{red}}$ b) Free energy change of electron transfer calculated using $\Delta \mathrm{G}=\mathrm{E}_{\mathrm{ox}}-$ $\mathrm{E}_{\mathrm{red}}-\mathrm{E}_{00}$, using potentials vs NHE.

Table S3. $\mathrm{H}_{2}$ production data for photocathodes

\begin{tabular}{|c|c|c|c|c|c|c|}
\hline Substrate & Dye & Catalyst & Condition & $\begin{array}{c}\text { Charge / } \\
\text { mC }\end{array}$ & $\mathrm{H}_{2} / \mathrm{nmol}$ & $\begin{array}{c}\text { Faradaic } \\
\text { efficiency / \% }\end{array}$ \\
\hline $\mathrm{NiO}$ & RBG & CoHEC & MES pH 5.5 & 100 & $376^{\mathrm{a}}$ & 73 \\
\hline $\mathrm{NiO}$ & RBG & CoHEC & MES pH 5.5 & 88 & $317^{\mathrm{a}}$ & 69 \\
\hline $\mathrm{NiO}$ & RBG & CoHEC & MES pH 5.5 & 109 & 288 & 49 \\
\hline $\mathrm{NiO}$ & RBG & CoHEC & $\begin{array}{c}\text { Na phosphate } \\
\text { pH } 7\end{array}$ & 124 & $461^{\mathrm{a}}$ & 72 \\
\hline $\mathrm{NiO}$ & RBG & CoHEC & $\begin{array}{c}\text { Na phosphate } \\
\text { pH } 7\end{array}$ & 135 & $618^{a}$ & 88 \\
\hline $\mathrm{NiO}$ & RBG & $\begin{array}{l}\text { CoHEC } \\
\text { ligand }\end{array}$ & MES pH 5.5 & 56 & 15 & 7 \\
\hline $\mathrm{NiO}$ & P1 & & MES pH 5.5 & 31 & 0 & 0 \\
\hline $\begin{array}{c}\mathrm{NiO} \text { (no } \\
\text { ozone) }\end{array}$ & RBG & CoHEC & MES pH 5.5 & 49 & 12 & 7 \\
\hline $\mathrm{CuGaO}_{2}$ & RBG & CoHEC & $\begin{array}{c}\text { Na phosphate } \\
\text { pH } 7\end{array}$ & 145 & 555 & 74 \\
\hline
\end{tabular}

a) Measurement of headspace and solution

\section{$\underline{\text { STH calculations }}$}

This work: Z-Scheme of $\mathrm{CuGaO}_{2}|\mathbf{R B G}-174| \mathrm{CoHEC}$ and $\mathrm{CoO}_{\mathbf{x}} / \mathrm{TaON}$

Using Equation 1 in the manuscript.

Average current $=10.3 \mu \mathrm{A}$

Potential to split water $=1.23 \mathrm{~V}$

Faradaic efficiency $=87 \%$

Total incident light $($ cathode + anode $)=204.9 \mathrm{~mW}$

$$
\mathrm{STH}=\frac{0.0103 \mathrm{~mA} \times 87 \% \times 1.23 \mathrm{~V}}{204.9 \mathrm{~mW}}=5.4 \times 10^{-3} \%
$$


Z-Scheme reported in Sun and coworkers, J. Am. Chem. Soc, 2015, 137, 9153-9159

Steady current read from Figure $\mathrm{S} 15=15 \mu \mathrm{A}$

Potential to split water $=1.23 \mathrm{~V}$

Faradaic efficiency $=55 \%$

Total incident light $($ cathode + anode $)=200 \mathrm{~mW}$

$$
\mathrm{STH}=\frac{0.015 \mathrm{~mA} \times 55 \% \times 1.23 \mathrm{~V}}{200 \mathrm{~mW}}=5.1 \times 10^{-3} \%
$$

